\title{
The Björling problem for non-minimal constant mean curvature surfaces
}

\author{
DAVID Brander AND Josef F. Dorfmeister
}

\begin{abstract}
The classical Björling problem is to find the minimal surface containing a given real analytic curve with tangent planes prescribed along the curve. We consider the generalization of this problem to non-minimal constant mean curvature (CMC) surfaces, and show that it can be solved via the loop group formulation for such surfaces. The main result gives a way to compute the holomorphic potential for the solution directly from the Björling data, using only elementary differentiation, integration and holomorphic extensions of real analytic functions. Combined with an Iwasawa decomposition of the loop group, this gives the solution, in analogue to Schwarz's formula for the minimal case. Some preliminary examples of applications to the construction of CMC surfaces with special properties are given.
\end{abstract}

\section{Introduction}

In this article we consider the following:

Problem 1.1. Let $J \subset \mathbb{R}$ be an open interval. Let $f_{0}: J \rightarrow \mathbb{E}^{3}$ be a regular real analytic curve, with tangent vector field $f_{0}^{\prime}$. Let $v: J \rightarrow \mathbb{E}^{3}$ be a non-vanishing analytic vector field along $f_{0}$ such that the inner product $\left\langle v, f_{0}^{\prime}\right\rangle=0$ along the curve. Let $H$ be a non-zero real number. Find all conformal constant mean curvature (CMC) H immersions, $f: \Sigma \rightarrow E^{3}$, where $\Sigma$ is some open subset of $\mathbb{C}$ containing $J$, such that the restriction $\left.f\right|_{J}$ coincides with $f_{0}$, and such that the tangent planes to the immersion along $f_{0}$ are spanned by $v$ and $f_{0}^{\prime}$.

This generalizes, to the case $H \neq 0$, Björling's problem for minimal surfaces, proposed by E.G. Björling in 1844 and solved by H.A. Schwarz in 1890. See, for example,[5].

In the minimal case, there is a simple formula for the surface involving nothing but integrals and analytic continuation of the initial data: specifically, if $f_{0}(x)$ is as above, and $n(x)$ is the unit normal to the prescribed 
family of tangent planes, then, letting $\check{n}(z)$ and $\check{f}_{0}(z)$ be the analytic extensions of these functions away from the curve, Schwarz's formula for the unique solution to the Björling problem is

$$
f(x, y)=\Re\left\{\check{f}_{0}(z)-\mathrm{i} \int_{x_{0}}^{z} \check{n}(w) \wedge d \check{f}_{0}(w)\right\} .
$$

The Schwarz formula has been an important tool in the study of minimal surfaces: as a means to prove general facts about the surfaces, such as the fact that if a minimal surface intersects a plane perpendicularly, then this plane is a plane of symmetry of the surface. It has been especially useful for constructing explicit examples of minimal surfaces; for example, with certain symmetries (see [5]). For recent examples of applications to global problems of minimal surfaces see $[8,12]$.

The existence of the Schwarz formula is connected to the fact that the Gauss map of a minimal surface is holomorphic, and to the Weierstrass representation for minimal surfaces. Moreover, the Björling problem has also been studied in some other situations, for example in works by Gálvez, Mira and collaborators $[1,9,10]$, where it is called the geometric Cauchy problem. These geometric problems all have Weierstrass representations in terms of holomorphic data, analogous to the case of minimal surfaces.

On the other hand, when $H \neq 0$ the Gauss map is merely harmonic, rather than holomorphic.

\subsection{Results of this article}

1.1.1. Solution of the Björling problem. In this article, we make use of the so-called generalized Weierstrass representation for CMC surfaces of Dorfmeister et al.[7] to show that the Björling problem can be solved for non-minimal CMC surfaces. The main result is Theorem 3.1 which gives a method for computing the (unique) solution in terms of the given data $f_{0}$ and $v$, using elementary integration and differentiation, analytic continuation of real functions and an Iwasawa factorization of the loop group. In fact, our construction is highly analogous to the Schwarz formula given above: we take the loop group extended frame for the Gauss map determined by the family of planes along the curve, extend this holomorphically away from the curve and then apply an Iwasawa decomposition of the complex loop group, to obtain the "real part" of the complexified frame. This turns out to be an extended frame for the Gauss map of the desired surface, and the Sym-Bobenko formula retrieves the CMC surface. 
A point that is perhaps not obvious in the procedure just described is the fact that the loop group frame, which is defined in terms of the Hopf differential and the metric of the surface (not the Gauss map alone), can actually be constructed given only the Björling data on a curve.

As a side benefit, Theorem 3.1 also gives a new way to compute the holomorphic data, a so-called holomorphic potential, which determine a given CMC surface, using nothing but analytic continuation and integration. We call this new holomorphic potential a boundary potential, because it is an analytic extension of the Maurer-Cartan form of the (real) extended frame for the surface in question, which is computed along a curve from the Björling data.

The construction of this boundary potential differs in an important respect from a previous method given by $\mathrm{Wu}$ [18] for finding the normalized meromorphic potential for a CMC surface, because Wu's formula uses the holomorphic part of the function $u$ - where the metric is given by $4 \mathrm{e}^{2 u}\left(d x^{2}+d y^{2}\right)-$ and this cannot be determined directly from the Björling data along a curve.

1.1.2. Two-parameter families of CMC surfaces. A new feature for the Björling problem arises when one considers the non-minimal case: namely, one now has the constant $H$ entering into the construction, which can take any non-zero real value, and hence can be thought of as a parameter. Thus, in the non-minimal case, the solution to the Björling problem actually gives a family of CMC surfaces through the given curve, varying continuously with $H$. In Example 3.1, by taking the curve to be the unit circle in the $x_{1} x_{2^{-}}$ plane and $v$ to point in the $x_{3}$ direction, we obtain a family of potentials, representing a deformation of a sphere (minus two points, strictly speaking) through the Delaunay surfaces and the cylinder, all containing the same circle.

This leads to a corollary, Theorem 3.2 which says that, given a CMC-1 surface $f$ and some choice of conformal parameterization, with metric $4 \mathrm{e}^{2 u}$ $\left(d x^{2}+d y^{2}\right)$, there is a continuous one-parameter family of CMC-1 surfaces $f^{t}$, where $t \in \mathbb{R}$, such that $f^{1}=f$, and the Hopf differential of $f^{t}$ is $Q_{t} d z^{2}=$ $\left(2(1-t) \mathrm{e}^{2 \check{u}_{0}}+Q\right) d z^{2}$, where $\check{u}_{0}$ is a holomorphic extension of $\left.u\right|_{J}$, and $J$ is a given curve in the parameter domain. This family is different from that associated to the loop parameter, which scales the Hopf differential by a complex constant.

1.1.3. Applications to boundary value problems and to the construction of CMC surfaces with symmetries. The experience of the use of Schwarz's formula in the study of minimal surfaces indicates that 
Theorem 3.1 should be a useful tool for the non-minimal case. We consider some preliminary examples in Section 4.

Concerning boundary value problems, the problem of finding a CMC surface with a given boundary curve $\gamma$ can always be formulated as a Björling problem, provided that $\gamma$ is a real analytic curve. This is because every CMC surface admits a conformal parameterization, and, if the boundary is real analytic, the surface can be extended over the boundary [13]. Now given such a curve $f_{0}$, one can consider all possible vector fields $v$ for the Björling problem, and thus finds expressions for the boundary potentials of all possible solutions. In Section 4 we consider the simplest case of an open curve, and characterize all CMC surfaces that contain a straight line, the $x_{1}$-axis, in terms of the angle made between the normal to the surface along the line and the $x_{3}$-axis. Theorem 4.1 gives the formula for the boundary potential for such a surface; we then use the software CMCLab [16] to exhibit examples with particular properties, such as the first two surfaces shown in figure 1.

The case that the boundary curve is closed, although more complicated in general, can also be studied using this approach. In the final section, we construct some examples of surfaces that contain a planar circle (figures 1 right, 4 to 6$)$.

\section{The loop group formulation and DPW method}

In this section, we summarize standard facts about CMC surfaces and their construction via integrable systems methods. The loop group formulation for CMC surfaces in Euclidean space $\mathbb{E}^{3}$ evolved from the work of Sym [17], Pinkall and Sterling [14] and Bobenko [2, 4]. The Sym-Bobenko formula for CMC surfaces was given by Bobenko [3, 4], similar to the formula for constant negative Gauss surfaces of Sym [17]. The DPW method for constructing all CMC surfaces from holomorphic data is due to Dorfmeister
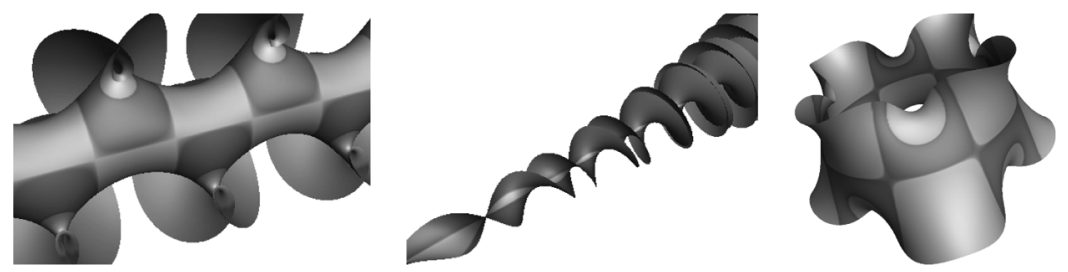

Figure 1: Two conformally immersed non-minimal CMC surfaces which contain an entire straight line, and one which contains a planar circle. The surfaces are colored according to Gaussian curvature. 
et al. [7]. We give only an outline of the DPW construction here, without reference to its more general purpose as a method for constructing pluriharmonic maps into symmetric spaces.

\subsection{The $S U(2)$-frame for a conformal immersion}

It is known that every CMC surface admits a conformal parameterization. Therefore, we first describe a standard SU(2) frame for a conformally parameterized surface. For the Lie algebra $\mathfrak{s u}(2)$, we work with the basis

$$
e_{1}=\left(\begin{array}{cc}
0 & -\mathrm{i} \\
-\mathrm{i} & 0
\end{array}\right), \quad e_{2}=\left(\begin{array}{cc}
0 & 1 \\
-1 & 0
\end{array}\right), \quad e_{3}=\left(\begin{array}{cc}
\mathrm{i} & 0 \\
0 & -\mathrm{i}
\end{array}\right) \text {. }
$$

We identify Euclidean three-space $\mathbb{E}^{3}$ with $\mathfrak{s u}(2)$, with inner product given by $\langle X, Y\rangle=-\frac{1}{2} \operatorname{trace}(X Y)$, giving the orthonormal relations $\left\langle e_{i}, e_{j}\right\rangle=\delta_{i j}$.

Let $\Sigma$ be a connected Riemann surface, and suppose $f: \Sigma \rightarrow \mathbb{E}^{3}$ is a conformal immersion with (not necessarily constant) mean curvature $H$. Conformality means we can choose coordinates $z=x+\mathrm{i} y$ and define a function $u: \Sigma \rightarrow \mathbb{R}$ such that the metric is given by

$$
\mathrm{d} s^{2}=4 e^{2 u}\left(\mathrm{~d} x^{2}+\mathrm{d} y^{2}\right) .
$$

A frame $F: \Sigma \rightarrow \mathrm{SU}(2)$ is uniquely determined up to multiplication by \pm 1 by the conditions

$$
F e_{1} F^{-1}=\frac{f_{x}}{\left|f_{x}\right|}, \quad F e_{2} F^{-1}=\frac{f_{y}}{\left|f_{y}\right|} .
$$

The sign ambiguity is removed by choosing coordinates for $\mathbb{E}^{3}$ so that the frame $F$ satisfies $F\left(z_{0}\right)=I$, for some fixed point $z_{0}$.

A choice of unit normal vector is given by $N=\mathrm{Fe}_{3} F^{-1}$. The Hopf differential is defined to be $Q \mathrm{~d} z^{2}$, where

$$
Q:=\left\langle N, f_{z z}\right\rangle
$$

The Maurer-Cartan form, $\alpha$, for the frame $F$ is defined by

$$
\alpha:=F^{-1} \mathrm{~d} F=U \mathrm{~d} z+V \mathrm{~d} \bar{z} .
$$


The mean curvature is $H=\frac{1}{8} \mathrm{e}^{-2 u}\left\langle f_{x x}+f_{y y}, N\right\rangle$, and we have the formulae: $f_{z z}=2 u_{z} f_{z}+Q N, f_{\bar{z} \bar{z}}=2 u_{\bar{z}} f_{\bar{z}}+\bar{Q} N, f_{z \bar{z}}=2 H e^{2 u} N$ and

$$
f_{z}=-2 \mathrm{ie}^{u} F\left(\begin{array}{ll}
0 & 1 \\
0 & 0
\end{array}\right) F^{-1} \quad f_{\bar{z}}=-2 \mathrm{ie}^{u} F\left(\begin{array}{ll}
0 & 0 \\
1 & 0
\end{array}\right) F^{-1} .
$$

Differentiating these, one obtains the following:

Lemma 2.1. The connection coefficients $U:=F^{-1} F_{z}$ and $V:=F^{-1} F_{\bar{z}}$ are given by

$$
U=\frac{1}{2}\left(\begin{array}{cc}
u_{z} & -2 H \mathrm{e}^{u} \\
Q \mathrm{e}^{-u} & -u_{z}
\end{array}\right), \quad V=\frac{1}{2}\left(\begin{array}{cc}
-u_{\bar{z}} & -\bar{Q} \mathrm{e}^{-u} \\
2 H \mathrm{e}^{u} & u_{\bar{z}}
\end{array}\right) .
$$

The compatibility condition $d \alpha+\alpha \wedge \alpha=0$ is equivalent to the pair of equations

$$
\begin{aligned}
& u_{z \bar{z}}+H^{2} \mathrm{e}^{2 u}-\frac{1}{4}|Q|^{2} \mathrm{e}^{-2 u}=0, \\
& Q_{\bar{z}}=2 \mathrm{e}^{2 u} H_{z} .
\end{aligned}
$$

\subsection{CMC surfaces, the loop group and the Sym-Bobenko formula}

Now suppose we insert a parameter $\lambda$ into the 1-form $\alpha$, defining the family $\hat{\alpha}:=\hat{U} d z+\hat{V} d \bar{z}$, where

$$
\hat{U}=\frac{1}{2}\left(\begin{array}{cc}
u_{z} & -2 H \mathrm{e}^{u} \lambda^{-1} \\
Q \mathrm{e}^{-u} \lambda^{-1} & -u_{z}
\end{array}\right), \quad \hat{V}=\frac{1}{2}\left(\begin{array}{cc}
-u_{\bar{z}} & -\bar{Q} \mathrm{e}^{-u} \lambda \\
2 H \mathrm{e}^{u} \lambda & u_{\bar{z}}
\end{array}\right) .
$$

The loop group characterization for CMC surfaces is contained in the following fact, which is quickly verified by adding $\lambda$ at the appropriate places in the compatibility conditions (2.5) above:

Lemma 2.2. The one-form $\hat{\alpha}$ satisfies the Maurer-Cartan equation

$$
d \hat{\alpha}+\hat{\alpha} \wedge \hat{\alpha}=0
$$

for all $\lambda \in \mathbb{C} \backslash\{0\}$ if and only if the following two conditions both hold:

(1) $[d \hat{\alpha}+\hat{\alpha} \wedge \hat{\alpha}]_{\lambda=1}=0$,

(2) the mean curvature $H$ is constant. 
Note that it also follows from (2.5) that the Hopf differential for a CMC surface is holomorphic.

Now $\hat{\alpha}$ is a one-form with values in the Lie algebra $\operatorname{Lie}\left(\Lambda G_{\sigma}\right)$, where $\Lambda G_{\sigma}$ is the loop group of maps from the unit circle $\mathbb{S}^{1}$ into $G=\mathrm{SU}(2)$, with a twisting condition that amounts to diagonal and off-diagonal components being, respectively, even and odd functions in the $\mathbb{S}^{1}$-parameter $\lambda$. The condition that the Maurer-Cartan equation is satisfied for all $\lambda$ means that $\hat{\alpha}(\cdot, \lambda)$ can be integrated for every $\lambda$ to obtain a map $\hat{F}: \widetilde{\Sigma} \rightarrow \Lambda G_{\sigma}$ from the universal cover of $\Sigma$ into $\Lambda G_{\sigma}$.

Definition 2.1. The map $\hat{F}: \widetilde{\Sigma} \rightarrow \Lambda G_{\sigma}$ obtained by integrating the above one-form $\hat{\alpha}$, with the initial condition $\hat{F}\left(z_{0}\right)=I$, is called an extended frame for the CMC surface $f$.

Note that $\left.\hat{F}\right|_{\lambda=1}: \tilde{\Sigma} \rightarrow \mathrm{SU}(2)$ coincides with the original frame $F$.

If $H$ is any non-zero real constant, the Sym-Bobenko formula, at $\lambda \in \mathbb{S}^{1}$, is given by

$$
\mathcal{S}_{\lambda}(\hat{F}):=-\frac{1}{2 H}\left(\hat{F} e_{3} \hat{F}^{-1}+2 \mathrm{i} \lambda \partial_{\lambda} \hat{F} \hat{F}^{-1}\right)
$$

Note: Setting $\lambda=\mathrm{e}^{\mathrm{i} t}$, we have $\frac{\partial}{\partial t}=\mathrm{i} \lambda \frac{\partial}{\partial \lambda}$. Hence $\mathcal{S}_{\lambda}(\hat{F})$ takes values in $\mathfrak{s u}(2)=$ $\mathbb{E}^{3}$.

Theorem 2.1 (3, 4]). (1) Given a CMC H surface, $f$, with extended frame $\hat{F}: \widetilde{\Sigma} \rightarrow \Lambda G_{\sigma}$, described above, the original surface $f$ is recovered by the formula

$$
f(x, y)=\mathcal{S}_{1}(\hat{F}(z))-\mathcal{S}_{1}\left(\hat{F}\left(z_{0}\right)\right)+f\left(z_{0}\right)
$$

For other values $\lambda_{0} \in \mathbb{S}^{1}, \mathcal{S}_{\lambda_{0}}(\hat{F}): \tilde{\Sigma} \rightarrow \mathbb{E}^{3}$ is also a CMCH surface in $\mathbb{E}^{3}$, with Hopf differential given by $\lambda_{0}^{-2} Q$.

(2) Conversely, given a map $\hat{F}: \tilde{\Sigma} \rightarrow \Lambda G_{\sigma}$, the Maurer-Cartan of which has coefficients of the form given by $(2.6)$, the map $\mathcal{S}_{\lambda_{0}}(\hat{F}): \tilde{\Sigma} \rightarrow \mathbb{E}^{3}$ obtained by the Sym-Bobenko formula is a CMC H immersion into $\mathbb{E}^{3}$.

(3) If $D(z)$ is any diagonal matrix valued function, constant in $\lambda$, then $\mathcal{S}_{\lambda}(\hat{F} D)=\mathcal{S}_{\lambda}(\hat{F})$.

Proof. For item (1), set $\hat{f}^{\lambda}:=\mathcal{S}_{\lambda}(\hat{F})$ then compute that $\hat{f}_{z}^{1}=f_{z}$ and $\hat{f}_{\bar{z}}^{1}=$ $f_{\bar{z}}$, so $f$ and $\hat{f}^{1}$ are the same surface up to translation. Formula (2.8) 
follows immediately. For other values of $\lambda$, see item (2). To prove (2), one computes $\hat{f}_{z}^{\lambda_{0}}$ and $\hat{f}_{\bar{z}}^{\lambda_{0}}$, and then the metric, the Hopf differential and the mean curvature. Item (3) of the theorem is obvious.

\subsection{The DPW construction}

Let $\Lambda G_{\sigma}^{\mathbb{C}}$ denote the group of loops in $G^{\mathbb{C}}=\operatorname{SL}(2, \mathbb{C})$ with the twisting described above. Let $\Lambda^{+} G_{\sigma}^{\mathbb{C}}$ denote the subgroup of loops which extend holomorphically to the unit disc. For the purpose of normalizations, we also use the subgroup

$$
\Lambda_{P}^{+} G_{\sigma}^{\mathbb{C}}:=\left\{B \in \Lambda^{+} G_{\sigma}^{\mathbb{C}} \mid B(0)=\left(\begin{array}{cc}
\rho & 0 \\
0 & \rho^{-1}
\end{array}\right), \rho \in \mathbb{R}, \rho>0\right\}
$$

In order to describe the DPW method, we need the following standard loop group decomposition, which allows one to write a $G^{\mathbb{C}}$-valued loop as a product of a $G$-valued loop and a loop that extends holomorphically to the unit disc.

Theorem 2.2 ([7, 15]; The Iwasawa Decomposition). Any element $g$ of $\Lambda G_{\sigma}^{\mathbb{C}}$ can be uniquely expressed as a product

$$
g=F B, \quad F \in \Lambda G_{\sigma}, \quad B \in \Lambda_{P}^{+} G_{\sigma}^{\mathbb{C}}
$$

The factors $F$ and $B$ depend real analytically on $g$.

We can now state the theorem of Dorfmeister, Pedit and Wu which is fundamental to what follows. An explicit example is given below.

Theorem 2.3 ([6, 7]; Generalized Weierstrass representation for CMC surfaces in $\left.\mathbb{E}^{3}\right)$. Let

$$
\begin{aligned}
& \hat{\xi}=\sum_{i=-1}^{\infty} A_{i} \lambda^{i} d z \in \operatorname{Lie}\left(\Lambda G_{\sigma}^{\mathbb{C}}\right) \otimes \Omega^{1,0}(\Sigma) \\
& A_{-1}=\left(\begin{array}{cc}
0 & a_{-1} \\
b_{-1} & 0
\end{array}\right), \quad a_{-1} \text { non-vanishing }
\end{aligned}
$$

be a holomorphic one-form over a simply connected Riemann surface $\Sigma$. 
Let $\hat{\Phi}: \Sigma \rightarrow \Lambda G_{\sigma}^{\mathbb{C}}$ be a solution of

$$
\hat{\Phi}^{-1} d \hat{\Phi}=\hat{\xi}
$$

Consider the unique decomposition obtained from applying Theorem 2.2 pointwise on $\Sigma$ :

$$
\hat{\Phi}=\hat{F} \hat{B}, \quad \hat{F}: \Sigma \rightarrow \Lambda G_{\sigma}, \quad \hat{B}: \Sigma \rightarrow \Lambda_{P}^{+} G_{\sigma}^{\mathbb{C}}
$$

Then for any $\lambda_{0} \in \mathbb{S}^{1}$, the map $\mathcal{S}_{\lambda_{0}}(\hat{F}): \Sigma \rightarrow \mathbb{E}^{3}$ given by the Sym-Bobenko formula (2.7), is a conformal CMC H immersion.

Conversely, let $\Sigma$ be a non-compact Riemann surface. Then any nonminimal conformal CMC immersion from $\Sigma$ into $\mathbb{E}^{3}$ can be constructed in this manner, using a holomorphic potential $\hat{\xi}$ that is well defined on $\Sigma$.

To prove the first direction in Theorem 2.3, one can show, using the fact that $\hat{\xi}=\lambda^{-1} A_{-1}+\cdots$ and that $\hat{F}$ is unitary, that we can write

$$
\hat{F}^{-1} d \hat{F}=\left(\begin{array}{cc}
c & a \lambda^{-1} \\
b \lambda^{-1} & -c
\end{array}\right) d z+\left(\begin{array}{cc}
-\bar{c} & -\bar{b} \lambda \\
-\bar{a} \lambda & \bar{c}
\end{array}\right) d \bar{z} .
$$

Setting $f=\mathcal{S}_{\lambda_{0}} \hat{F}$, one then computes that

$$
\hat{F}^{-1} f_{z} \hat{F}=\left(\begin{array}{cc}
0 & -4 \mathrm{i} a \lambda_{0}^{-1} \\
0 & 0
\end{array}\right), \quad \hat{F}^{-1} f_{\bar{z}} \hat{F}=\left(\begin{array}{cc}
0 & 0 \\
-4 \mathrm{i} \bar{a} \lambda_{0} & 0
\end{array}\right) .
$$

Post-multiplying the frame $\hat{F}$ by a diagonal matrix which is independent of $\lambda$ (and therefore does not change $f$ ), we can assume that $a \lambda_{0}^{-1}$ is real and positive, and write $a \lambda_{0}^{-1}=\bar{a} \lambda_{0}=\frac{1}{2} \mathrm{e}^{u}$. Then, comparing $\hat{F}^{-1} f_{z} \hat{F}$ and $\hat{F}^{-1} f_{\bar{z}} \hat{F}$ with (2.3), it follows that $f$ is conformally immersed and $\hat{F}$ is the coordinate frame defined in Section 2.1. Moreover, the above expression (2.10) for the Maurer-Cartan form of $\hat{F}$ means that $\hat{F}$ satisfies the requirements of Theorem 2.1 to be the frame for a CMC surface. The condition $a_{-1} \neq 0$ is the regularity condition, and it is also true that the surface has umbilics at points where $b_{-1}=0$.

The map $\hat{\Phi}$ above is called a holomorphic extended frame for $f$. Note that a holomorphic extended frame is by no means unique: however, if we allow meromorphic frames, then there is a unique frame where $\hat{\xi}$ is of the form $A_{-1} \lambda^{-1} d z$. 
Example 2.1 (A cylinder). Let

$$
\hat{\xi}=\left(\begin{array}{cc}
0 & \lambda^{-1} d z \\
\lambda^{-1} d z & 0
\end{array}\right)
$$

on $\Sigma=\mathbb{C}$. Then one solution $\hat{\Phi}$ of $d \hat{\Phi}=\hat{\Phi} \hat{\xi}$ is

$$
\hat{\Phi}=\exp \left\{\left(\begin{array}{cc}
0 & z \lambda^{-1} \\
z \lambda^{-1} & 0
\end{array}\right)\right\}
$$

which has the Iwasawa splitting $\hat{\Phi}=\hat{F} \hat{B}$, where

$$
\hat{F}=\exp \left\{\left(\begin{array}{cc}
0 & z \lambda^{-1}-\bar{z} \lambda \\
z \lambda^{-1}-\bar{z} \lambda & 0
\end{array}\right)\right\}, \quad \hat{B}=\exp \left\{\left(\begin{array}{cc}
0 & \bar{z} \lambda \\
\bar{z} \lambda & 0
\end{array}\right)\right\},
$$

take values in $\Lambda G_{\sigma}$ and $\Lambda_{P}^{+} G_{\sigma}^{\mathbb{C}}$ respectively. The Sym-Bobenko formula $\mathcal{S}_{1}(\hat{F})$ gives the surface

$$
\frac{-1}{2 H}[4 x, \sin (4 y), \cos (4 y)]
$$

in $\mathbb{E}^{3}=\left\{\left[x_{1}, x_{2}, x_{0}\right]:=x_{1} e_{1}+x_{2} e_{2}+x_{0} e_{3}\right\}$.

\section{Solution of the Björling problem via the DPW method}

We are now ready to consider Problem 1.1. We are given a real analytic function $f_{0}: J \rightarrow \mathbb{E}^{3}$, which we want to extend to a conformally immersed CMC surface $f: \Sigma \rightarrow \mathbb{E}^{3}$, where $\Sigma$ is some open subset of $\mathbb{C}$ containing the set $J \times\{0\}$, which we also denote by $J$. Such an extension is not unique, but we are also given the tangent plane to the surface along the image of $J$, in the form of a regular real analytic unit vector field $v: J \rightarrow \mathbb{E}^{3}$, such that $\left\langle v, \frac{d f_{0}}{d x}\right\rangle=0$. The required surface is to be tangent to the plane spanned by $\frac{d f_{0}}{d x}$ and $v$ along the curve.

If an extension exists, then we can choose an extended frame $\hat{F}: \Sigma \rightarrow$ $\Lambda G_{\sigma}$, as described above, such that $f$ is given by the Sym-Bobenko formula $\mathcal{S}_{1}(\hat{F})$. We will construct $\hat{F}$ (and hence $f$ ) from the boundary data given by $f_{0}, v$.

The idea is that it will be enough to find the Maurer-Cartan form of $F$ in terms of the matrices $U$ and $V$ in (2.4) only on the interval $J$. Then we can insert the parameter $\lambda$ as in (2.6), integrate this to find an expression, 
$\hat{F}_{0}$, for $\hat{F}$ along $J$. Then, it turns out, the holomorphic extension of this will be a holomorphic extended frame $\hat{\Phi}$ for the surface we seek.

Examining expression (2.4) for $U$ and $V$, namely:

$$
U=F^{-1} F_{z}=\frac{1}{2}\left(\begin{array}{cc}
u_{z} & -2 H \mathrm{e}^{u} \\
Q \mathrm{e}^{-u} & -u_{z}
\end{array}\right), \quad V=F^{-1} F_{\bar{z}}=\frac{1}{2}\left(\begin{array}{cc}
-u_{\bar{z}} & -\bar{Q} \mathrm{e}^{-u} \\
2 H \mathrm{e}^{u} & u_{\bar{z}}
\end{array}\right),
$$

we see that, in order to insert the parameter $\lambda$, and hence integrate to find the extended frame, it is necessary and sufficient to find the three functions:

$$
u, \frac{d u}{d z} \text { and } Q
$$

along $J$, and so this is the first goal.

The data $\frac{d f_{0}}{d x}$ and $v$ give us an $\mathrm{SU}(2)$-frame along $J$ as described in Section 2.1. Since we seek a conformal immersion, and $v$ is orthogonal to $\frac{d f_{0}}{d x}$, we require that $\frac{\partial f}{\partial y}=2 \mathrm{e}^{u} v$ along $J$, and our standard frame from (2.2) is determined (up to a sign), by

$$
F_{0} e_{1} F_{0}^{-1}=\frac{1}{2} \mathrm{e}^{-u} \frac{d f_{0}}{d x}, \quad F_{0} e_{2} F_{0}^{-1}=v
$$

where $u$ is yet to be determined. We can choose coordinates for $\mathbb{E}^{3}$ such that $\frac{d f_{0}}{d x}\left(x_{0}\right)$ and $v\left(x_{0}\right)$ point in the directions of $e_{1}$ and $e_{2}$ respectively, so that $F_{0}\left(x_{0}\right)=I$.

Now, by definition of $F_{0}$, it is necessary that

$$
\frac{d f_{0}}{d x}=2 \mathrm{e}^{u} F_{0}\left(\begin{array}{cc}
0 & -\mathrm{i} \\
-\mathrm{i} & 0
\end{array}\right) F_{0}^{-1}
$$

and, taking the determinant, we obtain the formula

$$
u=\ln \left(\frac{1}{2} \sqrt{\operatorname{det}\left(\frac{d f_{0}}{d x}\right)}\right),
$$

which can also be deduced by the requirement that $\left\langle\frac{d f_{0}}{d x}, \frac{d f_{0}}{d x}\right\rangle=4 \mathrm{e}^{2 u}$.

Now differentiating our frame $F_{0}$ along $J$ with respect to the parameter $x$, we can write

$$
F_{0}^{-1}\left(F_{0}\right)_{x}=\left(\begin{array}{cc}
a & b \\
-\bar{b} & -a
\end{array}\right)
$$


where both $a$ and $b$ are known functions of $x$, and $a$ is pure imaginary. According to Lemma 2.1, the extension $F$ of $F_{0}$ away from $J$ must satisfy

$$
\begin{aligned}
F^{-1} F_{x} & =U+V \\
& =\frac{1}{2}\left(\begin{array}{cc}
u_{z}-u_{\bar{z}} & -2 H \mathrm{e}^{u}-\bar{Q} \mathrm{e}^{-u} \\
Q \mathrm{e}^{-u}+2 H \mathrm{e}^{u} & -u_{z}+u_{\bar{z}}
\end{array}\right),
\end{aligned}
$$

and this must agree along $J$ with expression (3.2) corresponding to $F_{0}$. The $(1,1)$ components give

$$
u_{z}-u_{\bar{z}}=2 a \text {. }
$$

On the other hand, we have, by definition,

$$
u_{z}+u_{\bar{z}}=u_{x}
$$

Adding these equations gives

$$
u_{z}=a+\frac{1}{2} u_{x}
$$

in terms of known functions along $J$.

Now we use the $(1,2)$ components of the matrices above to get

$$
Q=-2 \mathrm{e}^{u}\left(\bar{b}+H \mathrm{e}^{u}\right) .
$$

We can find the extended frame $\hat{F}_{0}$ along $J$ by inserting these expressions for $u, u_{z}$ and $Q$ into the restriction of the one-form given by Equations (2.6) to the real line, namely

$$
\begin{aligned}
\hat{\alpha}_{0}= & \frac{1}{2}\left(\left(\begin{array}{cc}
0 & -2 H \mathrm{e}^{u} \\
Q \mathrm{e}^{-u} & 0
\end{array}\right) \lambda^{-1}+\left(\begin{array}{cc}
u_{z}-u_{\bar{z}} & 0 \\
0 & -u_{z}+u_{\bar{z}}
\end{array}\right)\right. \\
& \left.+\left(\begin{array}{cc}
0 & -\bar{Q} \mathrm{e}^{-u} \\
2 H \mathrm{e}^{u} & 0
\end{array}\right) \lambda\right) d x,
\end{aligned}
$$

and then integrating this along $J$ by solving the equation $\hat{F}_{0}^{-1} d \hat{F}_{0}=\hat{\alpha}_{0}$ with the initial condition $\hat{F}_{0}\left(x_{0}\right)=I$.

We can now state the main result of this article:

Theorem 3.1. Let $\hat{F}_{0}: J \rightarrow \Lambda G_{\sigma}$ be the extended frame along $J$ constructed above. Then

(1) there exists an open set $\Sigma \subset \mathbb{C}$ containing $J$, and a holomorphic map $\hat{\Phi}: \Sigma \rightarrow \Lambda G_{\sigma}^{\mathbb{C}}$ such that the restriction $\left.\hat{\Phi}\right|_{J}$ of $\hat{\Phi}$ to $J$ is equal to $\hat{F}_{0} ;$ 
(2) the Maurer-Cartan form of $\hat{\Phi}$ has a Fourier expansion in $\lambda$ :

$$
\hat{\Phi}^{-1} d \hat{\Phi}=\sum_{i=-1}^{1} A_{i} \lambda^{i} d z, \quad\left[A_{-1}\right]_{11} \neq 0 .
$$

(3) the surface $f: \Sigma \rightarrow \mathbb{E}^{3}$ obtained from $\hat{\Phi}$ via the pointwise Iwasawa decomposition $\hat{\Phi}=\hat{F} \hat{B}$, with $\hat{F} \in \Lambda G_{\sigma}$ and $\hat{B} \in \Lambda_{P}^{+} G_{\sigma}^{\mathbb{C}}$, followed by the Sym-Bobenko formula:

$$
f(x, y)=\mathcal{S}_{1}(\hat{F}(z))-\mathcal{S}_{1}\left(\hat{F}\left(z_{0}\right)\right)+f\left(z_{0}\right)
$$

is of constant mean curvature $H$, restricts to $f_{0}$ along $J$, and is tangent along $J$ to the plane spanned by $\frac{d f_{0}}{d x}$ and $v$;

(4) the surface $f$ so constructed is the unique solution to Problem 1.1, in the following sense: if $\tilde{f}$ is any other solution, then, for every point $x_{0} \in J$, there exists a neighborhood $\mathcal{N}=\left(x_{0}-\epsilon, x_{0}+\epsilon\right) \times(-\delta, \delta) \subset \mathbb{C}$ of $z_{0}=\left(x_{0}, 0\right)$ such that $\left.f\right|_{\mathcal{N}}=\left.\tilde{f}\right|_{\mathcal{N}}$.

Proof. Items (1) and (2): $\hat{F}_{0}$ is obtained by solving the equation $\hat{F}_{0}^{-1} d \hat{F}_{0}=$ $\hat{\alpha}_{0}=(\hat{U}+\hat{V}) d x$, with the initial condition $\hat{F}\left(x_{0}\right)=I$. Now $\hat{\alpha}_{0}$ is of the form (3.6). By construction, the components of the three coefficient matrices of $\hat{\alpha}_{0}$ are all real analytic along $J$. Hence there is some open set $\Sigma \subset \mathbb{C}$, containing $J$, to which they simultaneously extend holomorphically. Since the component $\left[\left(\hat{\alpha}_{0}\right)_{-1}\right]_{11}=-H \mathrm{e}^{u}$ is non-vanishing on $J$, we can arrange, by choosing $\Sigma$ sufficiently small, that this also holds for the holomorphic extension. Substituting these holomorphic extensions for their counterparts, and $d z$ for $d x$, into the expression above for $\hat{\alpha}_{0}$ gives a holomorphic extension $\hat{\alpha}$ of $\hat{\alpha}_{0}$. Since $\hat{\alpha}$ has trace zero and is twisted, this holomorphic one-form takes values in the Lie algebra $\operatorname{Lie}\left(\Lambda G_{\sigma}^{\mathbb{C}}\right)$. We can choose $\Sigma$ to be contractible, and then the equation $\hat{\Phi}^{-1} d \hat{\Phi}, \hat{\Phi}\left(z_{0}\right)=I$ can be solved uniquely to obtain the required map $\hat{\Phi}: \Sigma \rightarrow \Lambda G_{\sigma}^{\mathbb{C}}$.

Item (3): That the CMC surface $f: \Sigma \rightarrow \mathbb{E}^{3}$ exists is assured by Theorem 2.3 , since $\hat{\Phi}^{-1} d \hat{\Phi}$ has the required form. Now the surface $f$ is obtained by taking the unique Iwasawa decomposition

$$
\hat{\Phi}=\hat{F} \hat{B}
$$

where $\hat{F} \in \Lambda G_{\sigma}$, and $B \in \Lambda_{P}^{+} G_{\sigma}^{\mathbb{C}}$, and applying the Sym-Bobenko formula to $\hat{F}$. Since $\left.\hat{\Phi}\right|_{J}=\hat{F}_{0}$, which takes values in $\Lambda G_{\sigma}$, it follows that the splitting 
along $J$ is just $\hat{\Phi}=\hat{F}_{0} I$. In other words, $\left.\hat{F}\right|_{J}=\hat{F}_{0}$. Hence $\mathcal{S}_{1}\left(\left.\hat{F}\right|_{J}\right)=\mathcal{S}_{1}\left(\hat{F}_{0}\right)$, and this is shown to be equal to $f_{0}$ by a computation, as in the proof of Theorem 2.1. The fact that $f$ is tangent along $J$ to the plane spanned by $\frac{d f_{0}}{d x}$ and $v$ is built into the construction of the frame $F_{0}$ along $J$.

Item (4): for uniqueness, it is enough to show locally that any two CMC $H$ surfaces that are equal and tangent along a part of a curve are the same surface. This can be done by a maximal principle, or can also be seen from the construction of $\hat{F}_{0}$ given here. About any point $x_{0} \in J$, we have given a canonical means to construct a unique extended frame $\hat{F}_{0}$ along $J$, with $\hat{F}_{0}\left(x_{0}\right)=I$. Now use the Birkhoff decomposition [15] of $\Lambda G_{\sigma}^{\mathbb{C}}$ to write

$$
\hat{F}_{0}(x)=\hat{F}_{0}^{-} \hat{F}_{0}^{+}
$$

where $\hat{F}_{0}^{+} \in \Lambda^{+} G_{\sigma}^{\mathbb{C}}$, and $\hat{F}_{0}^{-}$is a loop that extends holomorphically to the exterior of the unit disc in the Riemann sphere and is normalized so that $\hat{F}_{0}^{-}(\lambda=\infty)=I$. This can be done pointwise on an open subset of $J$ containing $x_{0}$ because $\hat{F}_{0}\left(x_{0}\right)$ is in the big cell. Then $\hat{F}_{0}^{-}$is uniquely determined by $\hat{F}_{0}$, depends real analytically on $x$ (see $[7]$ ), and, it is straightforward to verify, has a Maurer-Cartan form of a very simple form:

$$
\left(\hat{F}_{0}^{-}\right)^{-1} d \hat{F}_{0}^{-}=\left(\begin{array}{cc}
0 & a_{0} \\
b_{0} & 0
\end{array}\right) \lambda^{-1} d x, \quad a_{0} \neq 0 .
$$

The real analytic functions $a_{0}$ and $b_{0}$ have unique holomorphic extensions $a$ and $b$ to some neighborhood of $\left(x_{0}, 0\right)$ in $\mathbb{C}$, and putting these into the one-form

$$
\hat{\xi}=\left(\begin{array}{ll}
0 & a \\
b & 0
\end{array}\right) \lambda^{-1} d z
$$

we see that we have a potential for a CMC surface, as in Theorem 2.3.

On the other hand, if we are given two surfaces that solve the Björling problem, we could just as well have constructed the extended frame for each of the two surfaces on some neighborhood of the point $z_{0}=\left(x_{0}, 0\right)$ in $\mathbb{C}$, rather than restricting to the real line. For each surface we obtain a unique map $\tilde{F}^{-}$, with $\tilde{F}^{-}(\lambda=\infty)=I$, and a Maurer-Cartan form of the form (3.8). One can verify that this so-called normalized potential is holomorphic, and, since the corresponding holomorphic functions $\tilde{a}$ and $\tilde{b}$ agree, by construction, with $a_{0}$ and $b_{0}$ along $J$, it follows that they agree everywhere and the surface constructed from $\hat{\xi}$ is the original surface. Hence the two original surfaces are the same. 
Definition 3.1. The holomorphic extension $\hat{\alpha}$ of $\hat{\alpha}_{0}$ defined in the proof above will be called the boundary potential for the CMC surface in question.

\subsection{Example}

As a test case, we compute the solution when the initial curve is a circle in a plane, and the tangent plane along the curve is orthogonal to this plane.

We take the circle

$$
f_{0}(x)=[\sin 2 x, 0,-\cos 2 x]=\left(\begin{array}{cc}
-i \cos 2 x & -i \sin 2 x \\
-i \sin 2 x & \mathrm{i} \cos 2 x
\end{array}\right),
$$

and the vector field

$$
v(y)=[0,1,0]=\left(\begin{array}{cc}
0 & 1 \\
-1 & 0
\end{array}\right)
$$

Then $\frac{d f_{0}}{d x}(x)=2 \mathrm{i}\left(\begin{array}{cc}\sin 2 x & -\cos 2 x \\ -\cos 2 x & -\sin 2 x\end{array}\right)$, and using expression (3.1) we must have

$$
u=\ln \left(\frac{1}{2} \sqrt{\operatorname{det}\left(\frac{d f_{0}}{d x}\right)}\right)=0 .
$$

To find $a$ and $b$ in $F_{0}^{-1}\left(F_{0}\right)_{x}$, we need to convert the vector fields $\frac{d f_{0}}{d x}$ and $v$ into an $\mathrm{SU}(2)$-frame $F_{0}(x)$. The vector fields in question are orthogonal, so, we look for a conformal immersion with coordinates $z=x+\mathrm{i} y$ and such that $f_{y}$ is in the direction of $v$. The frame according to the recipe is determined by

$$
F_{0} e_{1} F_{0}^{-1}=\frac{1}{2} \mathrm{e}^{-u} \frac{d f_{0}}{d x}, \quad F_{0} e_{2} F_{0}^{-1}=v
$$

Setting $F_{0}=\left(\begin{array}{cc}A & B \\ -\bar{B} & \bar{A}\end{array}\right)$, the first of these two equations gives

$$
2 \Re(A \bar{B})=-\sin 2 x, \quad A^{2}-B^{2}=\cos 2 x,
$$

and the second equation gives

$$
\Im(A \bar{B})=0, \quad A^{2}+B^{2}=1 .
$$


The unique solution that satisfies $F_{0}(0)=I$ is the $\mathrm{SU}(2)$-frame

$$
F_{0}(x)=\left(\begin{array}{cc}
\cos x & -\sin x \\
\sin x & \cos x
\end{array}\right) .
$$

Now we equate

$$
F_{0}^{-1}\left(F_{0}\right)_{x}=\left(\begin{array}{cc}
0 & -1 \\
1 & 0
\end{array}\right)=\left(\begin{array}{cc}
a & b \\
-\bar{b} & -a
\end{array}\right),
$$

so that $a=0$ and $b=-1$.

Substituting into Equations (3.4) and (3.5) we have, along the real axis,

$$
u_{z}=0, \quad Q=2(1-H),
$$

and the extended frame $\hat{F}_{0}$ along the $x$-axis is computed by integrating the Maurer-Cartan form

$$
\hat{\alpha}_{0}=\frac{1}{2}\left(\left(\begin{array}{cc}
0 & -2 H \\
2(1-H) & 0
\end{array}\right) \lambda^{-1}+\left(\begin{array}{cc}
0 & -2(1-H) \\
2 H & 0
\end{array}\right) \lambda\right) d x .
$$

Hence the boundary potential for the surface given by Theorem 3.1 is

$$
\hat{\alpha}(z)=\left(\begin{array}{cc}
0 & (H-1) \lambda-H \lambda^{-1} \\
H \lambda-(H-1) \lambda^{-1} & 0
\end{array}\right) d z .
$$

When $H \neq 1$, this holomorphic potential satisfies the conditions to be that of a Delaunay surface (see [11], where the fact that the rotation parameter is i $y$ rather than $x$ introduces a minus sign in the lower left corner). One obtains a sphere when $H=1$, a cylinder when $H=\frac{1}{2}$, and unduloids and nodoids for other values of $H$.

\subsection{Two parameter families of CMC surfaces}

Observe that in the previous example, if we simply drop the $\frac{1}{H}$ term in front of the Sym-Bobenko formula, we actually obtain a one-parameter family of CMC 1 surfaces, which deforms a sphere (minus two points) of radius 1 continuously through a family of Delaunay surfaces to a cylinder of radius $\frac{1}{2}$. Thus we see that if we are given a sphere as our initial object, and the circle in the sphere, then we obtain a one-parameter family of CMC-1 surfaces, by going through the Björling construction starting with this circle, the tangent plane to the sphere, inserting $H$ (now thought of as just a real parameter) 
into expression (3.5) for $Q$, constructing the boundary potential, and finally using the Sym-Bobenko formula without the $\frac{1}{H}$ factor. Clearly we can do this for any surface and any given curve in the surface, so we have the following corollary of Theorem 3.1:

Theorem 3.2. Let $\Sigma \subset \mathbb{C}$ be a simply connected domain, containing the origin, and with coordinates $z=x+\mathrm{i} y$. Suppose given a conformal $C M C-1$ immersion $f: \Sigma \rightarrow E^{3}$, with metric given by $4 \mathrm{e}^{2 u}\left(d x^{2}+d y^{2}\right)$. Let $\Sigma^{\prime} \subset \Sigma$ be any simply connected open subset to which the functions $u_{0}(x):=u(x, 0)$ and $\eta_{0}(x):=-\mathrm{i} u_{y}(x, 0)=\left(u_{z}-u_{\bar{z}}\right)(x, 0)$ extend holomorphically, and denote the holomorphic extensions by $\check{u}_{0}$ and $\check{\eta}_{0}$. Then there exists a continuous oneparameter family of conformal CMC-1 immersions $f^{t}: \Sigma^{\prime} \rightarrow \mathbb{E}^{3}$, with Hopf differential given by

$$
Q_{t}=2(1-t) \mathrm{e}^{2 \check{u}_{0}}+Q
$$

and such that $f^{1}=\left.f\right|_{\Sigma^{\prime}}$. The surfaces are related on the real coordinate axis by the relation

$$
f^{t}(x, 0)=t f^{1}(x, 0) .
$$

The boundary potential for $f^{t}$ is

$$
\hat{\alpha}^{t}=\left(\begin{array}{cc}
\frac{1}{2} \check{\eta}_{0} & -t \mathrm{e}^{\check{u}_{0}} \lambda^{-1}-\left((1-t) \mathrm{e}^{\check{u}_{0}}+\frac{1}{2} \bar{Q} \mathrm{e}^{-\check{u}_{0}}\right) \lambda \\
\left((1-t) \mathrm{e}^{\check{u}_{0}}+\frac{1}{2} Q \mathrm{e}^{-\check{u}_{0}}\right) \lambda^{-1}+t \mathrm{e}^{\check{u}_{0}} \lambda & -\frac{1}{2} \check{\eta}_{0}
\end{array}\right) d z .
$$

Proof. The proof is a matter of going through the construction above for the solution of the Björling problem, starting with $f_{0}=f(x, 0)$. We have, for Equation (3.3)

$$
F^{-1} F_{x}=\frac{1}{2}\left(\begin{array}{cc}
u_{z}-u_{\bar{z}} & -2 \mathrm{e}^{u}-\bar{Q} \mathrm{e}^{-u} \\
Q \mathrm{e}^{-u}+2 \mathrm{e}^{u} & -u_{z}+u_{\bar{z}}
\end{array}\right),
$$

where $Q$ and $u$ are those of the given surface $f$ along the real axis, so that $b=-\mathrm{e}^{u}-\frac{1}{2} \bar{Q} \mathrm{e}^{-u}$, and substituting this into expression (3.5), and the parameter $t$ instead of $H$, we obtain the above expression for $Q_{t}$. Finally, we construct the surface from Theorem 3.1, and scale the result by a factor $t$, so that our surface is CMC 1 , rather than CMC $t$.

Note that this can be done for any open curve in the coordinate domain of a surface, by changing conformal coordinates so that this curve becomes a part of the $x$-axis. 


\section{Applications to boundary value problems and surfaces with symmetries}

By a result of F. Müller (Theorem 5 of [13]), given a conformally parameterized CMC surface with boundary, which is continuous at the boundary, and where the boundary curve in $\mathbb{E}^{3}$ is an embedded real analytic curve, the surface can be extended analytically across the boundary. Therefore, for such boundary curves, we may always assume that the boundary is contained in the surface, and have the possibility of treating it as a Björling problem, by considering all possible tangent planes along this curve.

\subsection{CMC surfaces which contain a line segment}

In this section we use the boundary potential to describe all simply connected CMC surfaces that contain a given line or line segment.

Theorem 4.1. Let $J=(\alpha, \beta)$ be an open interval, where we allow $\alpha=-\infty$ and $\beta=\infty$. Let $l=(2 \alpha, 2 \beta) \times\{0\} \times\{0\} \subset \mathbb{E}^{3}$.

(1) Given a real analytic function $\theta_{0}: J \rightarrow \mathbb{R}$, with $\theta_{0}\left(x_{0}\right)=0$ for some $x_{0} \in J$, let $\Sigma$ be any simply connected domain in $\mathbb{C}$, containing $J \times$ $\{0\}$, to which $\frac{d \theta_{0}}{d x}$ extends analytically. Denote this analytic extension by $\check{\theta}_{x}$. Then, for any real $H \neq 0$, there is a conformally parameterized CMC H surface $f: \Sigma \rightarrow E^{3}$ which maps $(\alpha, \beta) \times\{0\} \subset \mathbb{C}$ to l, via the map $(x, 0) \mapsto[2 x, 0,0]$. The Hopf differential of this surface is given by $Q d z$, where

$$
Q=-\mathrm{i} \check{\theta}_{x}-2 H
$$

The boundary potential of the surface is given by

$$
\hat{\alpha}=\left(\begin{array}{cc}
0 & -H \lambda^{-1}+\left(-\frac{1}{2} \mathrm{i} \check{\theta}_{x}+H\right) \lambda \\
\left(-\frac{1}{2} \mathrm{i} \check{\theta}_{x}-H\right) \lambda^{-1}+H \lambda &
\end{array}\right) d z
$$

(2) Conversely, any simply connected non-minimal CMC surface in $\mathbb{E}^{3}$ which contains the segment $l$, can be represented, on an open subset containing $l$, this way. For each $x \in J$, the value $\theta_{0}(x) \bmod 2 \pi$ is the angle between the normal to the surface at the point $[2 x, 0,0]$ and some fixed line in the plane spanned by $e_{2}$ and $e_{3}$. 
Proof. Item (1): This amounts to interpreting the function $\theta_{0}$ as the vector field $v$ for the Björling problem for the map $f_{0}: J \rightarrow \mathbb{E}^{3}$,

$$
f_{0}(x)=[2 x, 0,0]=\left(\begin{array}{cc}
0 & -2 \mathrm{i} x \\
-2 \mathrm{i} x & 0
\end{array}\right) .
$$

Since $f_{0}$ is always tangent to the $x_{1}$-axis, the map $v: J \rightarrow E^{3}$ determined by $\theta_{0}$ via

$$
v(x)=\left[0, \cos \theta_{0}, \sin \theta_{0}\right]=\left(\begin{array}{cc}
\mathrm{i} \sin \theta_{0} & \cos \theta_{0} \\
-\cos \theta_{0} & -\mathrm{i} \sin \theta_{0}
\end{array}\right)
$$

is orthogonal to $\frac{d f_{0}}{d x}$ for all $x$. The normalization $\theta_{0}\left(x_{0}\right)=0$ is equivalent to choosing $v\left(x_{0}\right)=e_{2}=\left(\begin{array}{cc}0 & 1 \\ -1 & 0\end{array}\right)$, which will give us our standard normalization of the frame: $F_{0}\left(x_{0}\right)=I$.

According to Equation (3.1), we have $u=\ln \left(\frac{1}{2} \sqrt{\operatorname{det} \frac{d f_{0}}{d x}}\right)=0$, and solving Equations (3.9), we obtain the unique $\mathrm{SU}(2)$ frame mapping $x_{0}$ to the identity

$$
F_{0}=\left(\begin{array}{cc}
\cos \frac{\theta_{0}}{2} & -i \sin \frac{\theta_{0}}{2} \\
-i \sin \frac{\theta_{0}}{2} & \cos \frac{\theta_{0}}{2}
\end{array}\right)
$$

Hence

$$
F_{0}^{-1}\left(F_{0}\right)_{x}=-\frac{\mathrm{i}}{2} \frac{d \theta_{0}}{d x}\left(\begin{array}{ll}
0 & 1 \\
1 & 0
\end{array}\right)
$$

and formulae (3.1), (3.4) and (3.5) are

$$
u=u_{z}=0, \quad Q=-i \frac{d \theta_{0}}{d x}-2 H,
$$

which, extending holomorphically, gives the expression for the Hopf differential above. Finally, substituting these into expression (3.6) for $\hat{\alpha}_{0}$, and extending holomorphically, we obtain the above expression for the boundary potential $\hat{\alpha}$.

Item (2): For the converse, on an open set containing $l$, one can always choose conformal coordinates $(x, y)$ such that $f$ maps $J \times\{0\} \rightarrow l$ by the function $f((x, 0))=[2 x, 0,0]$. Fix a point $\left[2 x_{0}, 0,0\right] \in l$ and change coordinates of $\mathbb{E}^{3}$ so that $\frac{\partial f}{\partial y}\left(x_{0}, 0\right)$ is in the $e_{2}$ direction. Then the frame $F_{0}$ will be given as above, where $\theta_{0}$ is the angle between the normal direction and our fixed choice of $e_{3}$ direction. By Theorem 3.1, a non-minimal CMC surface is determined by its Björling data, so the Hopf differential and boundary potential stated above, are those of the original surface. 


\subsection{Examples containing a straight line}

If we choose $\theta_{0}$ to be constant, we of course get a cylinder, with boundary potential:

$$
\hat{\alpha}=\left(\begin{array}{cc}
0 & -H \lambda^{-1}+H \lambda \\
-H \lambda^{-1}+H \lambda & 0
\end{array}\right) d z .
$$

To check that this potential really does give a cylinder, observe that the holomorphic extended frame obtained by integrating $\hat{\alpha}$ is $\hat{\Phi}(z)=\exp \left(\left(-H \lambda^{-1}+\right.\right.$ $H \lambda) z A)$, where $A=\left(\begin{array}{ll}0 & 1 \\ 1 & 0\end{array}\right)$. Since this can be written as $\hat{\Phi}(z)=\exp \left(-H \lambda^{-1}\right.$ $z A) \cdot \exp (H \lambda z A)$ and the second matrix is in $\Lambda_{P}^{+} G_{\sigma}^{\mathbb{C}}$, the second factor has no effect on the term $\hat{F}$ in the Iwasawa decomposition $\hat{\Phi}=\hat{F} \hat{B}, \hat{F} \in \Lambda G_{\sigma}$, $\hat{B} \in \Lambda_{P}^{+} G_{\sigma}^{\mathbb{C}}$. Hence the surface obtained from this potential is the same as the one obtained from the potential $\xi=-H \lambda^{-1} A d z$, which was shown to be a cylinder in Example 2.1.

If we choose $\theta_{0}=2 x$, to obtain a surface the normal to which rotates about the line in a spiral (figure 2), the boundary potential is

$$
\hat{\alpha}=\left(\begin{array}{cc}
0 & -H \lambda^{-1}+(-\mathrm{i}+H) \lambda \\
(-\mathrm{i}-H) \lambda^{-1}+H \lambda & 0
\end{array}\right) d z .
$$

If we choose $\theta_{0}=x^{2}$, to obtain a surface the normal to which rotates with constantly increasing angular velocity about the line (figure 3 ), the boundary potential is

$$
\hat{\alpha}=\left(\begin{array}{cc}
0 & -H \lambda^{-1}+(-\mathrm{i} z+H) \lambda \\
(-\mathrm{i} z-H) \lambda^{-1}+H \lambda & 0
\end{array}\right) d z .
$$

If we choose $\theta_{0}=\frac{\pi}{8} \sin ^{2}(x)$, then we obtain a surface the normal to which maintains a small and periodic angle with the $x_{3}$ direction, along the line $l$
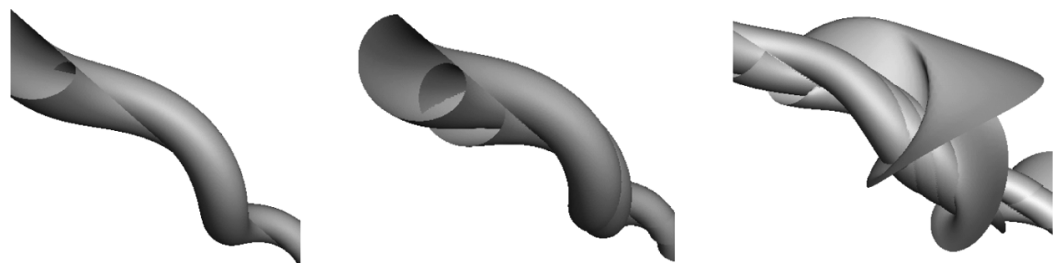

Figure 2: Three partial plots of one surface, containing a line $l$. The surface normal along the line $l$ rotates around $l$ at constant speed with respect to the arc-length parameter of $l$. 

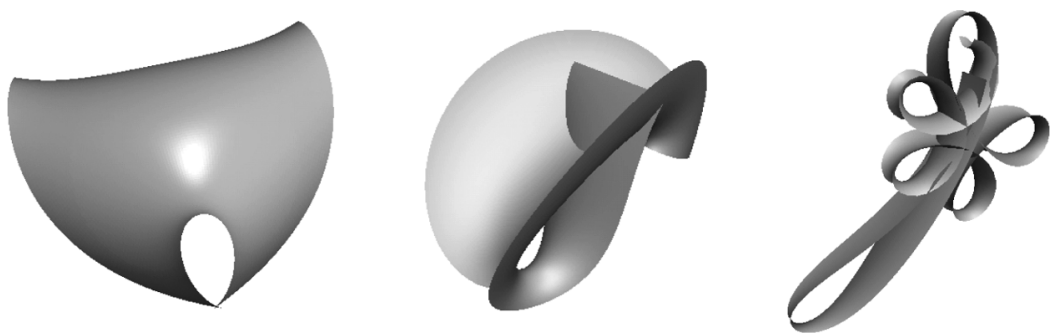

Figure 3: Three partial plots of a surface, containing a line $l$, the normal to which rotates with constantly increasing angular velocity around $l$. It is conformally parameterized by an immersion $f: \mathbb{C} \rightarrow \mathbb{E}^{3}$, which maps the real line to $l$. It has exactly one umbilic at $z=i$, around the spot of white light on the first image. The last two images show the image of a narrow strip around the positive imaginary axis. The image of a narrow strip around the positive real axis is shown in figure 1 (center).

(figure 1, first image). The boundary potential is $\hat{\alpha}=\left(\begin{array}{cc}0 & -H \lambda^{-1}+\left(-\mathrm{i} \frac{\pi}{8} \cos z \sin z+H\right) \lambda \\ \left(-\mathrm{i} \frac{\pi}{8} \cos z \sin z-H\right) \lambda^{-1}+H \lambda & 0\end{array}\right) d z$.

\subsection{Examples of CMC surfaces containing a planar circle}

Using an analogous approach for the circle, we can easily construct CMC surfaces containing a circle in a plane. The surfaces shown in figures 4 to 6 all come from boundary potentials of the form

$$
\alpha=\frac{1}{2}\left(\begin{array}{cc}
\stackrel{\frac{1}{z}(\cos (2 \check{\theta})-1)}{ }\left(H \lambda^{-1}+\left(\sin 2 \check{\theta}+H-2 \mathrm{i} \check{\theta}^{\prime}\right) \lambda\right. \\
\frac{1}{z^{2}}\left(\sin 2 \check{\theta}+H+2 \mathrm{i} \check{\theta}^{\prime}\right) \lambda^{-1}-\frac{1}{z^{2}} H \lambda & \frac{1}{z}(\cos (2 \check{\theta})-1)
\end{array}\right) d z,
$$
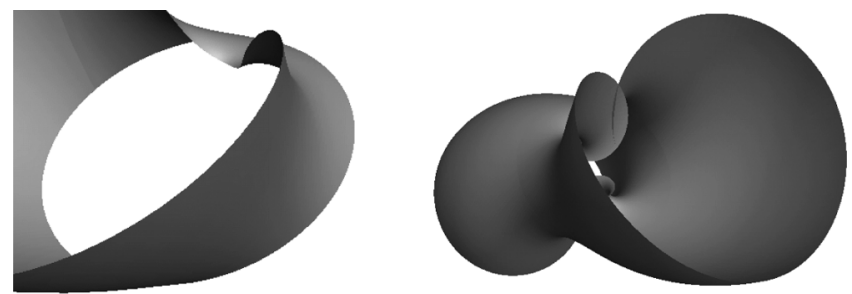

Figure 4: This surface contains a planar circle and has a reflective symmetry about the origin. 

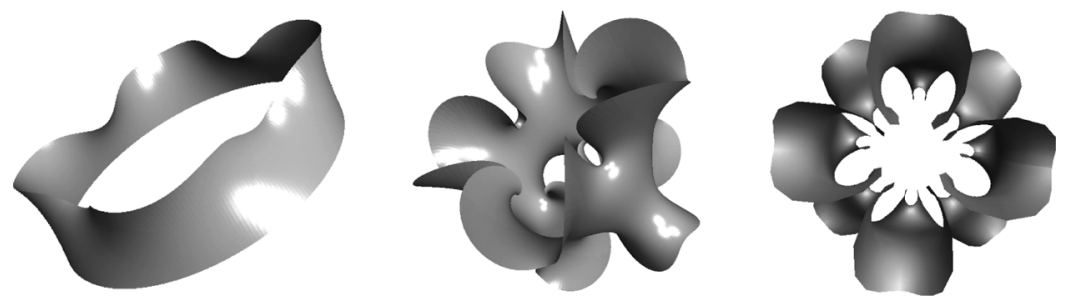

Figure 5: A CMC surface invariant under rotations of $\pi / 2$ around the $x_{3}$-axis.
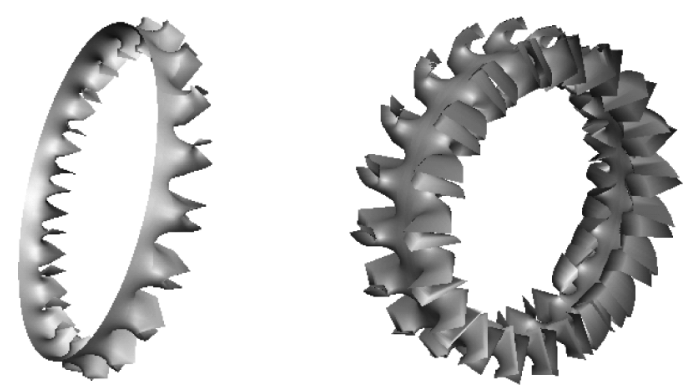

Figure 6: A CMC surface containing a planar circle and which is invariant under rotations of $\pi / 10$ around the $x_{3}$-axis.

where $\theta: \mathbb{R} \rightarrow \mathbb{R}$ satisfies $\theta(t+2 \pi)=\theta(t)+2 k \pi$ for some integer $k$, and $\check{\theta}$ is the analytic extension of $\theta(-\mathrm{i} \ln z)$ to an annulus around $S^{1}$. For Example 1, we used $\theta(t)$, up to a translation, proportional to $\sin (t)$; for the other examples $\theta(t)$ is, also up to a translation, proportional to $\sin ^{2}(k t)$ for some integer $k$.

\section{References}

[1] J. Aledo, R. Chaves and J. Gálvez, The Cauchy problem for improper affine spheres and the Hessian one equation, Trans. Amer. Math. Soc. 359 (2007), 4183-4208.

[2] A.I. Bobenko, All constant mean curvature tori in $R^{3}, S^{3}, H^{3}$ in terms of theta-functions, Math. Ann. 290 (1991), 209-245.

[3] A.I. Bobenko, Surfaces of constant mean curvature and integrable equations, Uspekhi Mat. Nauk 46(4) (1991), 3-42 (Translation in: Russian Math. Surveys 46 (1991), 1-45). 
[4] A.I. Bobenko, Surfaces in terms of 2 by 2 matrices. Old and new integrable cases, Harmonic Maps and Integrable systems, Aspects Math., E23, Vieweg, Braunschweig, 1994, 83-127.

[5] U. Dierkes, S. Hildebrandt, A. Küster and O. Wohlrab, Minimal surfaces. I. Boundary value problems, Grundlehren der Mathematischen Wissenschaften, 295, Springer-Verlag, Berlin, 1992.

[6] J. Dorfmeister and G. Haak, Construction of non-simply connected CMC surfaces via dressing, J. Math. Soc. Japan 55 (2) (2003), 335-364.

[7] J. Dorfmeister, F. Pedit, and H Wu, Weierstrass type representation of harmonic maps into symmetric spaces, Comm. Anal. Geom. 6 (1998), 633-668.

[8] J. Gálvez and P. Mira, Dense solutions to the Cauchy problem for minimal surfaces, Bull. Braz. Math. Soc. (N.S.) 35 (2004), 387-394.

[9] J. Gálvez and P. Mira, The Cauchy problem for the Liouville equation and Bryant surfaces, Adv. Math. 195 (2005), 456-490.

[10] J. Gálvez and P. Mira, Embedded isolated singularities of flat surfaces in hyperbolic 3-space, Calc. Var. Partial Differential Equations 24 (2005), 239-260.

[11] M. Kilian, On the associated family of Delaunay surfaces, Proc. Amer. Math. Soc. 132 (2004), 3075-3082.

[12] P. Mira, Complete minimal Möbius strips in $\mathbb{R}^{n}$ and the Björling problem, J. Geom. Phys. 56 (2006), 1506-1515.

[13] F. Müller, Analyticity of solutions for semilinear elliptic systems of second order, Calc. Var. Partial Differential Equations 15 (2002), $257-288$.

[14] U. Pinkall and I. Sterling, On the classification of constant mean curvature tori, Ann. of Math. (2) 130 (1989), 407-451.

[15] A. Pressley and G. Segal, Loop groups, Oxford Mathematical Monographs, Clarendon Press, Oxford, 1986.

[16] N. Schmitt, CMCLab, http://www.gang.umass.edu/software.

[17] A. Sym, Soliton surfaces and their applications, Geometric Aspects of the Einstein Equations and Integrable Systems, Lect. Notes Phys., 239, Springer, Berlin, 1985, 154-231. 
[18] H. Wu, A simple way for determining the normalized potentials for harmonic maps, Ann. Global Anal. Geom. 17 (1999), 189-199.

Department of Mathematics

Matematiktorvet, Building $303 \mathrm{~S}$

Technical University of Denmark

DK-2800 KGS. LyNGBY

DENMARK E-mail address: D.Brander@mat.dtu.dk

TU MÜNCHEN

Zentrum Mathematik (M8)

BOLTZMANNSTR. 3

85748 GARCHING

Germany

E-mail address: dorfm@ma.tum.de

Received September 25, 2009 